\title{
S100A6 promotes the proliferation and migration of cervical cancer cells via the PI3K/Akt signaling pathway
}

\author{
AIFANG LI, YUE GU, XUERU LI, HUI SUN, HE ZHA，JIAQING XIE，JIALI ZHAO, \\ MAO HUANG, LU CHEN, QI PENG, YAN ZHANG, YAGUANG WENG and LAN ZHOU \\ Key Laboratory of Medical Diagnostics, Ministry of Education, College of Laboratory Medicine, \\ Chongqing Medical University, Chongqing 400016, P.R. China
}

Received June 15, 2017; Accepted December 13, 2017

DOI: $10.3892 / \mathrm{ol} .2018 .8018$

\begin{abstract}
Cervical cancer is the second most common gynecological cancer worldwide and remains one of the leading causes of cancer-associated mortality among women. S100A6 has been reported to be associated with the development of many types of cancer. The aim of the present study was to investigate the effect of S100A6 on the proliferation, apoptosis and migration of cervical cancer cells and its underlying molecular mechanisms. Quantative polymerase chain reaction (qPCR) was used to detect the basic mRNA level of S100A6 in HeLa, SiHa and CaSki cells. Western blot analysis was used to detect the protein level of S100A6, epithelial cadherin, neuronal cadherin, phosphorylated protein kinase B (p-Akt), t-Akt, p-glycogen synthase kinase $3 \beta$ (GSK3 $\beta$ ), t-GSK $3 \beta$ and $\beta$-catenin. Semi-qPCR was used to detect the mRNA level of Snail, Twist and Vimentin. MTT and Hoechst staining assays were used to detect the proliferation and apoptosis of cells, and wound healing and Transwell assays were used to detect the migration of cells. The results of the present study demonstrate that the levels of S100A6 were decreased in HeLa cells compared with in $\mathrm{SiHa}$ and CaSki cells. Overexpression of S100A6 in HeLa and CaSki cells promoted the proliferative and migratory ability, and had no significant effect on cellular apoptosis. Whereas the knockdown of S100A6 in SiHa and CaSki cells inhibited the proliferative and migratory ability, it had no significant effect on apoptosis. The overexpression of S100A6 in HeLa cells increased the levels of neuronal $(\mathrm{N})$-cadherin, vimentin, Snail and Twist. Conversely, knockdown of S100A6 in SiHa cells decreased the levels of N-cadherin, vimentin, Snail and
\end{abstract}

Correspondence to: Dr Lan Zhou, Key Laboratory of Medical Diagnostics, Ministry of Education, College of Laboratory Medicine, Chongqing Medical University, 1 Yixueyuan Road, Yuzhong, Chongqing 400016, P.R. China

E-mail: zhoulan0111@foxmail.com

Key words: S100A6, cervical cancer, proliferation, migration, epithelial-mesenchymal transition, phosphoinositide 3-kinase/protein kinase B signaling pathway
Twist and increased the levels of epithelial (E)-cadherin. Furthermore, overexpression of S100A6 in HeLa cells activated the phosphoinositide 3-kinase (PI3K)/protein kinase B (Akt) signaling pathway, and treatment with the PI3K inhibitor LY294002 partially repressed S100A6-enhanced proliferation and migration of cervical cancer cells. These results indicate that S100A6 facilitates the malignant potential of cervical cancer cells, particularly metastatic ability and epithelial-mesenchymal transition, which is mediated by activating the PI3K/Akt signaling pathway.

\section{Introduction}

Cervical cancer originates from the epithelium of cervix uteri. It is one of the most common malignant tumors in women and the fourth leading cause of cancer-associated mortality worldwide (1). Persistent infection of high-risk human papillomaviruses (HPVs) is associated with the progression of cervical cancer (2). There have been a number of efficient measures for the diagnosis and prevention of cervical cancer, including screening programs and prophylactic HPV vaccine injections for patients with cervical cancer (3). Surgery, chemotherapy and radiotherapy are the primary therapies for cervical cancer; however, for patients with advanced cervical cancer, these methods are ineffective due to distant metastases. Consequently, the metastasis and recurrence of cervical cancer remain the principal causes of mortality. Therefore, there is a pressing requirement to elucidate the underlying molecular mechanisms, and to explore potential biomarkers and drug targets to develop novel options for the early diagnosis and treatment of cervical cancer.

S100A6 is a member of the S100 calcium-binding protein family and its gene is located at human chromosome 1q21, where chromosomal abnormalities occur frequently (4). Chromosome 1q21 polyploidy was considered a high-risk characteristic for patients with multiple myeloma receiving bortezomib therapy (5). Chromosome 1q21 abnormalities were also associated with intellectual disability, mild dysmorphic features and congenital heart disease (6). Previous studies have demonstrated that S100A6 is associated with tumorigenesis and tumor progression. S100A6 promotes the proliferation and migration of human colorectal cancer cells, hepatocellular carcinoma cells, osteosarcoma cells and 
epithelial-mesenchymal transition (EMT) of pancreatic cancer cells (7-10). Furthermore, S100A6 is associated with the poor prognosis of patients with gastric cancer (11). S100A6 has also been demonstrated to be involved in a number of signaling pathways such as phosphoinositide 3-kinase (PI3K)/protein kinase (Akt) (8), Wnt/ $\beta$-catenin $(10,12), \mathrm{p} 38 /$ mitogen-activated protein kinase (13) and nuclear factor- $\kappa \mathrm{B}$ signaling (14). However, to the best of our knowledge, there have not been any studies pertaining to the effect and underling molecular mechanism of S100A6 on cervical cancer at a cellular level.

The PI3K/Akt pathway is a key driver in carcinogenesis (15). Akt is a key signaling molecule that phosphorylates numerous downstream targets, connecting it to multiple interrelated signaling pathways. Thus, it is responsible for modulating several processes including cell survival, cell cycle progression, protein synthesis, angiogenesis and cellular migration (16-18). Akt is known to be overexpressed in many types of human cancer, and is associated with poor outcome in some cancer types (19). Therefore, it is hypothesized that the PI3K/Akt pathway is involved in progression of cervical cancer.

EMT is key regulator of metastasis as it facilitates tumor cell invasion and dissemination to distant organs. Standardized analysis of EMT markers in tumor biopsies may serve as a promising strategy for future clinical application (20). E-cadherin, N-cadherin, vimentin are EMT markers and Snail, Twist are EMT transcription factors and therefore the detection of these markers will reflect the status of EMT (20).

The aim of the present study was to investigate the effect of S100A6 on the proliferation and migration of cervical cancer cells and its underlying mechanisms. The present study hypothesizes that S100A6 exhibits the ability to promote the proliferation, migration and EMT of cervical cancer cells through activating PI3K/Akt signaling.

\section{Materials and methods}

Cell culture. The human cervical cancer cell lines HeLa, $\mathrm{SiHa}$ and CaSki were purchased from the American Type Culture Collection (ATCC; Manassas, VA, USA). Cells were maintained in Dulbecco's modified Eagle's medium (DMEM; HyClone; GE Healthcare Life Sciences, Logan, UT, USA) supplemented with $10 \%$ fetal bovine serum (FBS; Gibco; Thermo Fisher Scientific, Inc., Waltham, MA, USA) and incubated at $37^{\circ} \mathrm{C}$ with $5 \% \mathrm{CO}_{2}$.

Recombinant adenoviruses (Ad), recombinant proteins and reagents. AdS100A6 and AdsiS100A6 [carrying human S100A6 gene or S100A6-short interfering (si)RNA gene, respectively] and their controls [Ad-green fluorescent protein (GFP) or Ad-red fluorescent protein (RFP)] were gifts from Dr He (Medical Center, The University of Chicago, Chicago, IL, USA). Untreated cells were used as blank controls. Recombinant glutathione transferase (GST) and GST-S100A6 proteins were prepared according to the protocol of Miao et al (21). The following antibodies were used: Anti-human S100A6 (cat. no. sc-50409), anti- $\beta$-catenin (cat. no. sc-59737), anti-phosphorylated (p)-Akt (cat. no. sc-33437) and anti-total (t-)Akt (cat. no. sc-8312) (all from Santa Cruz Biotechnology, Inc., Dallas, TX, USA), anti-epithelial
(E)-cadherin (cat. no. 14472), anti-neuronal (N)-cadherin (cat. no. 13116), anti-p-glycogen synthase kinase $3 \beta$ (GSK3 $\beta$ ) (cat. no. 9323), anti-t-GSK3 $\beta$ (cat. no. 9315) (all from Cell Signaling Technology, Inc., Danvers, MA, USA), anti-E-cadherin for HeLa cells (cat. no. YM3353; ImmunoWay Biotechnology Company, Plano, TX, USA), anti- $\beta$-actin (cat. no. TA-09; OriGene Technologies, Inc., Beijing, China) and goat anti-rabbit antibody (cat. no. ZB-2301) or goat anti-mouse antibody (cat. no. ZB-2305) (OriGene Technologies, Inc.). MTT (Beyotime Biotechnology, Shanghai, China). Hoechst 33258 (Beyotime Institute of Biotechnology, Haimen, China) and the PI3K inhibitor LY294002 (MedChemExpress, Monmouth Junction, NJ, USA) were dissolved in dimethyl sulfoxide (DMSO) to a concentration of $10 \mathrm{mM}$.

RNA extraction, reverse transcription $(R T), q P C R$ and semi-qPCR. Total RNA was extracted from the cervical cancer cell lines using RNAiso Plus (Takara Biotechnology Co., Ltd., Dalian, China). RT reactions were performed according to the manufacturer's instructions (Takara Biotechnology Co., Ltd.). Semi-qPCR was performed according to the protocol of Duan et al (22). Taq DNA polymerase was purchased from Takara Biotechnology Co., Ltd., and the cDNA products were further diluted 5-fold and used in subsequent experiments. Cycling conditions were as follows: $94^{\circ} \mathrm{C}$ for $5 \mathrm{~min}, 94^{\circ} \mathrm{C}$ for $30 \mathrm{sec}, 68^{\circ} \mathrm{C}$ for $30 \mathrm{sec}$ and $72^{\circ} \mathrm{C}$ for 12 cycles with a decrease in $1^{\circ} \mathrm{C} /$ cycle; then, $94^{\circ} \mathrm{C}$ for $30 \mathrm{sec}, 55^{\circ} \mathrm{C}$ for $30 \mathrm{sec}$, and $72^{\circ} \mathrm{C}$ for $30 \mathrm{sec}$ for $18-27$ cycles depending on the abundance of the target genes. The PCR products were separated using $2 \%$ agarose gel and stained with ethidium bromide. The results were recorded using the Gel imaging system (GelDoc 1000; Bio-Rad Laboratories, Inc., Hercules, CA, USA) and analyzed using Quantity One (version 4.5.0; Bio-Rad Laboratories, Inc.).

qPCR was performed on the CFX96 real-time PCR detection system from Bio-Rad Laboratories, Inc. using SYBR Premix Ex Taq ${ }^{\mathrm{TM}}$ II (Takara Biotechnology Co., Ltd.). Data were collected and analyzed using the comparative $2^{-\Delta \Delta \mathrm{Ct}}$ method (23). GAPDH was used as control. All primers used in the present study are presented in Table I.

Western blot analysis. Total protein was extracted using radioimmunoprecipitation assay lysis buffer (Beyotime Biotechnology) in the presence of protease inhibitor (Complete EasyPack; Roche Applied Science, Penzberg, Germany) and phosphatase inhibitor (PhosSTOP; Roche Applied Science) at $72 \mathrm{~h}$ after treatment with recombinant adenoviruses or recombinant proteins. Protein was quantified using spectrophotometry (Thermo Fisher Scientific, Inc.) at $280 \mathrm{~nm}$. Protein (300 $\mu$ g loaded per lane) was separated using SDS-PAGE (10\% gel) and transferred onto polyvinylidene fluoride membranes (Sigma-Aldrich; Merck KGaA, Darmstadt, Germany). The membranes were blocked with $5 \%$ bovine serum albumin (Solarbio, Beijing, China) at $37^{\circ} \mathrm{C}$ for $2 \mathrm{~h}$ and incubated with primary antibodies (dilution 1:1,000) overnight at $4^{\circ} \mathrm{C}$. The membranes were washed with Tris-buffered saline with Tween-20 and incubated with horseradish peroxidase (HRP) conjugated secondary antibody (dilution 1:5,000) for $1 \mathrm{~h}$ at $37^{\circ} \mathrm{C}$. Finally, the membranes were exposed using Immobilon western chemiluminescent HRP substrate (EMD Millipore, Billerica, MA, USA). Results were recorded 
Table I. Primer sequences.

Gene

Primer sequence

Product size, bp

$\begin{array}{ll}\text { S100A6 } & \text { Forward, 5'-ATGGCATGCCCCTGGATCAGG-3' } \\ & \text { Reverse, 5'-TCAGCCCTTGAGGGCTTCAT-3' } \\ \text { Vimentin } & \text { Forward, 5'-TTGAACGCAAAGTGGAAT-3' } \\ & \text { Reverse, 5'-AGGTCAGGCTTGGAAACA-3' } \\ \text { Snail } & \text { Forward, 5'-ACCCCACATCCTTCTCACTG-3' } \\ & \text { Reverse, 5'-TACAAAAACCCACGCAGACA-3' } \\ \text { Twist } & \text { Forward, 5'-TCTTACGAGGAGCTGCAGAC-3' } \\ & \text { Reverse, 5'-TATCCAGCTCCAGAGTCTCT-3' } \\ \text { GAPDH } & \text { Forward, 5'-CAGCGACACCCACTCCTC-3' } \\ & \text { Reverse, 5'-TGAGGTCCACCACCCTGT-3' }\end{array}$

406

using the Bio-Rad electrophoresis documentation system (Gel Doc 1000; Bio-Rad Laboratories, Inc.) and Quantity One software (version 4.5.0; Bio-Rad Laboratories, Inc.).

Cell viability assay. Cell viability was determined using the MTT assay according to the protocol of Duan et al (7).

Hoechst staining assay. Cells were seeded ( $3 \times 10^{4}$ cells/well) in a 24-well plate and incubated at $37^{\circ} \mathrm{C}$ overnight, then treated with recombinant adenoviruses for $72 \mathrm{~h}$ when cells were $\sim 30 \%$ confluent. Cells were then fixed with $4 \%$ paraformaldehyde for $15 \mathrm{~min}$, washed with PBS three times, stained with $100 \mu \mathrm{l}$ Hoechst 33258 solution (dilution 1:1,000; Beyotime Institute of Biotechnology) for $30 \mathrm{~min}$ and washed with PBS a further three times. Images were captured under an inverted fluorescence microscope (magnification, x100 and x400). When the cell was apoptotic, the nucleus was stained bright blue. The ratio of the number of apoptotic cells to the total number of cells in a randomly given field of view was calculated as the rate of apoptosis.

Wound healing assay. Cells were seeded (3x10 cells/well) into a six-well plate and treated with recombinant adenoviruses for $72 \mathrm{~h}$ when cells reached $95 \%$ confluency. The wound healing assay was performed according to the protocol of Duan et al (7).

Transwell assay. The Transwell inserts were purchased from EMD Millipore. Cells were seeded $\left(1.5 \times 10^{5}\right.$ cells/well $)$ in a 6-well plate and treated with recombinant adenoviruses or recombinant proteins for $72 \mathrm{~h}$ when cells were $\sim 30 \%$ confluent. The treated cells were collected and $4 \times 10^{4}$ cells were resuspended in $400 \mu \mathrm{l}$ serum-free DMEM and seeded in the upper chamber, another $600 \mu \mathrm{l}$ medium with $20 \%$ FBS was added to the lower chamber. Following incubation for $24 \mathrm{~h}$, chambers were fixed with $4 \%$ paraformaldehyde for $15 \mathrm{~min}$ and then stained with $0.05 \%$ crystal violet for $15 \mathrm{~min}$ at room temperature. The transmembrane cells were counted using inverted microscopy (magnification, x100) in 10 different views for each insert and images were captured. The relative cell number per field was calculated as follows; number of cells in each group/number of cells in the blank group.
Statistical analysis. Results are presented as the mean \pm standard deviation. A one-way analysis of variance, followed by the Student-Newman-Keuls test was used to analyze the statistical significance of differences among groups, and all analyses were performed using GraphPad Prism software (version 5; GraphPad Software, Inc., La Jolla, CA, USA). $\mathrm{P}<0.05$ was considered to indicate a statistically significant difference.

\section{Results}

S100A6 promotes the proliferation of cervical cancer cells. To examine the effect of S100A6 on cervical cancer cells, the expression of S100A6 was detected in the three cervical cancer cell lines HeLa, SiHa and CaSki. The qPCR results demonstrated that the S100A6 mRNA level was comparatively lower in HeLa cells. As presented in Fig. 1A, the mRNA levels of S100A6 in SiHa and CaSki cells were increased 6.92- and 7.54-fold compared with those of HeLa cells respectively $(\mathrm{P}<0.005)$. The S100A6 level of HeLa and CaSki cells was upregulated by AdS100A6, and the S100A6 level of SiHa and CaSki cells was downregulated by AdsiS100A6 as was demonstrated by western blot analysis (Fig. 1B).

To investigate whether S100A6 is involved in the proliferation of cervical cancer cells, the MTT assay was performed. Cell proliferation ability was significantly increased following treatment with AdS100A6 for $72 \mathrm{~h}$ in HeLa and CaSki cells $(\mathrm{P}<0.01$ and $\mathrm{P}<0.005$ respectively; Fig. $1 \mathrm{C})$, whereas it was markedly decreased following treatment with AdsiS100A6 for $72 \mathrm{~h}$ in $\mathrm{SiHa}$ and CaSki cells $(\mathrm{P}<0.05$; Fig. 1D). The effect of S100A6 on cellular apoptosis was investigated using Hoechst staining and no marked changes were identified between the examined groups in number of apoptosis cells (Fig. 1E and F). These results demonstrated that S100A6 is able to promote the proliferation of cervical cancer cells and had no significant effect on the apoptosis of cells.

S100A6 promotes the migration and EMT of cervical cancer cells. Wound healing and Transwell assays were performed to investigate the effect of S100A6 on cell migration. In the present study, AdGFP was a control of AdS100A6 and AdRFP was a control of AdsiS100A6. Furthermore, CaSki cells were 


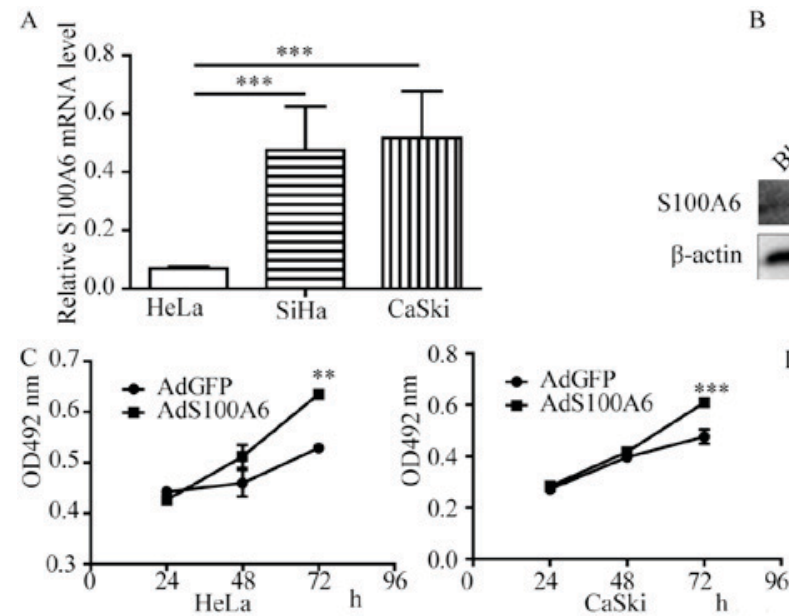

E

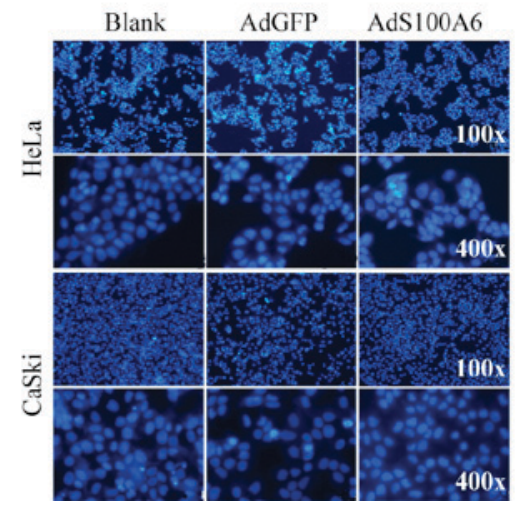

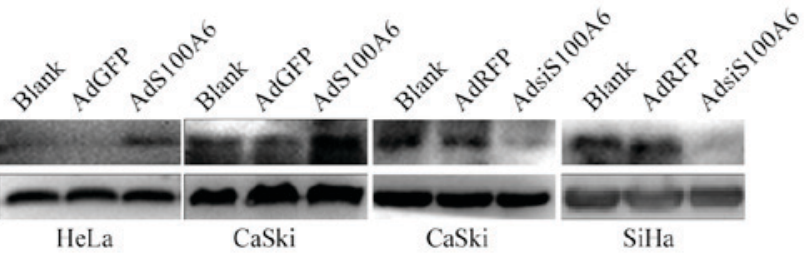
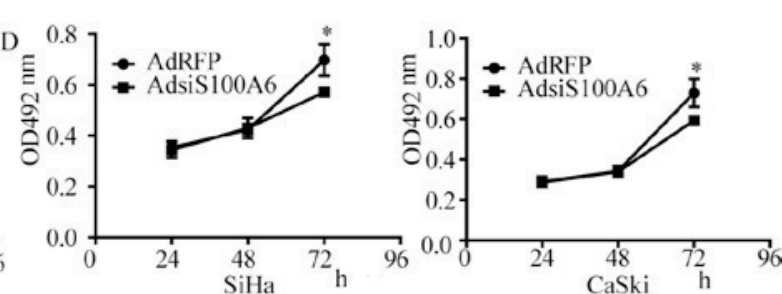

F

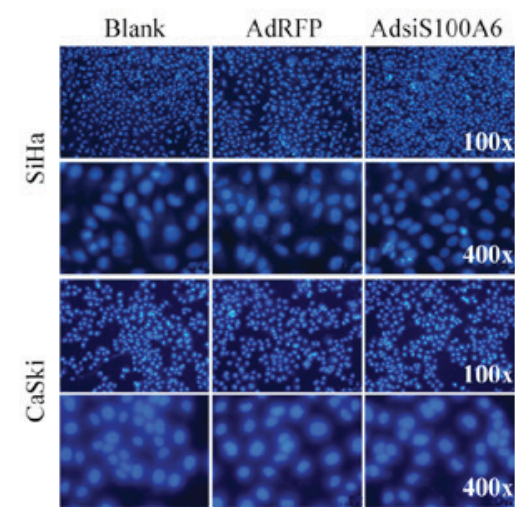

Figure 1. S100A6 promotes the proliferation of cervical cancer cells (A) qPCR was used to analyze the mRNA levels of S100A6 in the cervical cancer cell lines HeLa, SiHa and CaSki. (B) AdS100A6 infected HeLa and CaSki cells, AdsiS100A6 infected CaSki and SiHa cells. The infection efficiency was determined by western blotting. Cell proliferation was detected using an MTT assay in (C) HeLa and CaSki cells following treatment with AdS100A6 and in (D) SiHa and CaSki cells following treatment with AdsiS100A6. Cell apoptosis was determined by Hoechst staining assay in (E) HeLa and CaSki cells after treatment with AdS100A6 and in (F) SiHa and CaSki cells following treatment with AdsiS100A6. As no significant changes were identified between all groups in number of apoptosis cells, no quantitative data was provided. ${ }^{*} \mathrm{P}<0.05,{ }^{* *} \mathrm{P}<0.01,{ }^{* * *} \mathrm{P}<0.005$ compared with the AdGFP or AdRFP groups. All the experiments were repeated three times. Results were presented as the mean \pm standard deviation. A one-way analysis of variance, followed by the Student-Newman-Keuls test was used to analyze the statistical significance of differences among groups. OD, optical density; nm, nanometer; AdGFP, adenovirus carrying green fluorescent protein; AdS100A6, adenovirus carrying S100A6; AdRFP, adenovirus carrying red fluorescent protein; AdsiS100A6, adenovirus carrying S100A6-short interfering RNA gene; E-cadherin, epithelial cadherin; N-cadherin, neuronal cadherin.

treated with AdS100A6 and AdsiS100A6 in order to upregulate or downregulate the level of S100A6, respectively. The present study used AdS100A6 to upregulate the level of S100A6 in HeLa cells, and AdsiS100A6 to down-regulate the level of S100A6 in SiHa cells. Following the upregulation of S100A6 by AdS100A6 for $72 \mathrm{~h}$, the wound healing rates were increased 2.1- $(\mathrm{P}<0.05)$ and 3.6-fold $(\mathrm{P}<0.01)$ compared with those of the AdGFP groups in HeLa and CaSki cells (Fig. 2A). Conversely, following the downregulation of S100A6 by AdsiS100A6 for $72 \mathrm{~h}$, the wound-healing rates were decreased by $41 \%(\mathrm{P}<0.05)$ and $44 \%(\mathrm{P}<0.05)$ of that in their respective AdRFP groups (Fig. 2B). The migratory ability of cells was further confirmed by a Transwell assay. Following treatment with AdS100A6, the number of transmembrane cells were increased $\sim 5$ - $(\mathrm{P}<0.005)$ and 2 -fold $(\mathrm{P}<0.01)$ compared with that in the AdGFP groups of HeLa and CaSki cells (Fig. 2C). However, following treatment with AdsiS100A6, the number of transmembrane cells were decreased by $66 \%(\mathrm{P}<0.005)$ and $65 \%(\mathrm{P}<0.01)$ compared with those of their respective AdRFP groups (Fig. 2D). These results indicated that S100A6 promoted cell migration.

Additionally, upregulation of S100A6 in HeLa cells increased the mRNA levels of N-cadherin, vimentin, Snail and Twist, and decreased the protein level of E-cadherin, whereas the downregulation of S100A6 in SiHa cells induced the opposite effect (Fig. 2E). Collectively, these results indicated that S100A6 was able to induce EMT in cervical cancer cells.

S100A6 activates the PI3K/Akt signaling pathway and promotes the viability and migration of cervical cancer cells. To explore the predominant downstream signaling pathway of S100A6, the alteration of several pathway proteins were evaluated using western blotting. The phosphorylation level of Akt and its downstream target genes p-GSK3 $\beta$ and $\beta$-catenin were demonstrated to be increased in the AdS100A6 group of HeLa cells, and was consistent with activation of the PI3K/Akt pathway (Fig. 3A). To further validate the involvement of $\mathrm{PI} 3 \mathrm{~K} / \mathrm{Akt}$ activation in S100A6-enhanced proliferation and migration of cervical cancer cells, a pharmacological approach was adopted. HeLa cells were treated with the PI3K inhibitor LY294002 $(10 \mu \mathrm{M})$ alone, or combined with GST-S100A6 $(10 \mu \mathrm{g} / \mathrm{ml})$. The level of $\mathrm{p}-\mathrm{Akt}$, p-GSK3 $\beta$ and $\beta$-catenin were decreased when the PI3K/Akt pathway was blocked using the PI3K inhibitor LY294002 (Fig. 3B). Furthermore, LY294002 

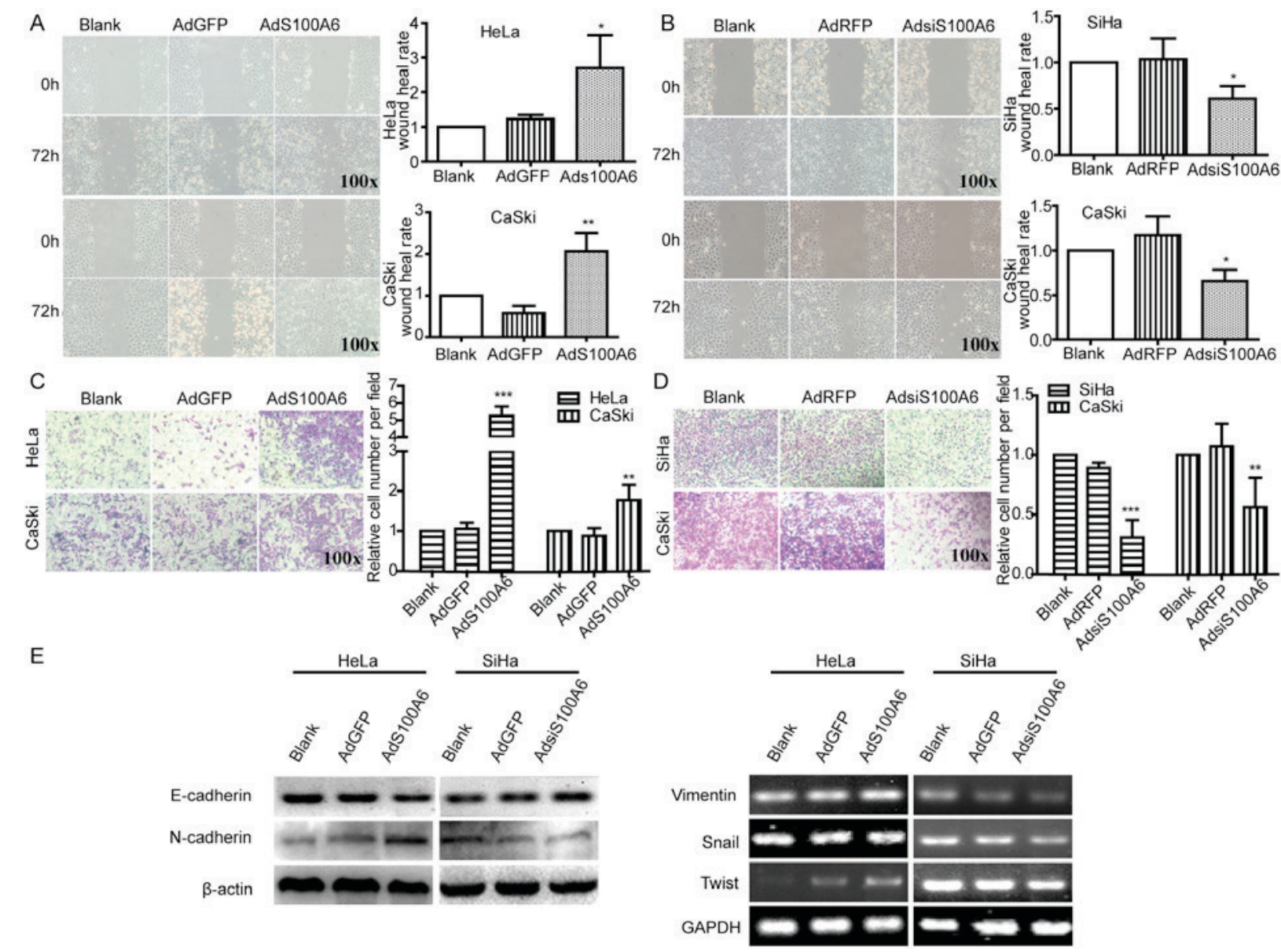

Figure 2. S100A6 promotes the migration and EMT of cervical cancer cells. Cell migration ability was detected using a wound healing assay. The wound width of sites was measured and the wound healing rate of (A) HeLa and CaSki cells with AdGFP, and (B) SiHa and CaSki with AdRFP was calculated. The wound healing rate was calculated as follows; (width at $0 \mathrm{~h}$-width at $72 \mathrm{~h}$ )/width at $0 \mathrm{~h} \times 100 \%$. Cell migration ability of (C) HeLa and CaSki cells with AdGFP, and (D) SiHa and CaSki cells with AdRFP were detected using a Transwell assay, where the number of transmembrane cells were counted and the relative cell number per field was calculated. The relative cell number per field = number of cells in per field of treatment group/number of cells in per field of blank group (E) The protein levels of E-cadherin and N-cadherin were determined using western blotting and the mRNA levels of vimentin, Snail and Twist were determined using semi-quantitative PCR in HeLa and SiHa cells. ${ }^{*} \mathrm{P}<0.05,{ }^{* *} \mathrm{P}<0.01,{ }^{* * *} \mathrm{P}<0.005$ compared with the AdGFP or AdRFP groups. All experiments were repeated for three times. Results were presented as the mean \pm standard deviation. A one-way analysis of variance, followed by the Student-Newman-Keuls test was used to analyze the statistical significance of differences among groups. EMT, epithelial-mesenchymal transition; AdGFP, adenovirus carrying green fluorescent protein; AdS100A6, adenovirus carrying S100A6; AdRFP, adenovirus carrying red fluorescent protein; AdsiS100A6, adenovirus carrying S100A6-short interfering RNA gene; E-cadherin, epithelial cadherin; N-cadherin, neuronal cadherin.

significantly impaired the S100A6-enhanced proliferation and migration of HeLa cells $(\mathrm{P}<0.005$; Fig. $3 \mathrm{C}$ and D). Taken together, these results suggest that S100A6 promotes the proliferation and migration of cervical cancer cells through the PI3K/Akt signaling pathway.

\section{Discussion}

Cervical cancer is a common tumor of the female reproductive system with a high metastatic potential and mortality. Although infection with HPV is considered to be a principal cause of cervical cancer, there are a variety of risk factors associated with the morbidity of cervical cancer, including reproductive and nutritional factors, and other types of pathogen infection including chlamydia trachomatis, herpes simplex virus type II and trichomonad. The presence of these additional risk factors provides additional difficulties in the identification of novel potential biomarkers for the detection and therapy of cervical cancer.
There have been a number of studies investigating the role of S100A6 in tumorigenesis and tumor development. The results provide evidence that $\mathrm{S} 100 \mathrm{~A} 6$ promotes proliferation and migration in colorectal cancer cells (7), pancreatic cancer cells (24) and other malignant tumor cells (9). A previous study indicated that the level of S100A6 differs in different cervical lesion tissues, and its expression is positively associated with the degree of cervical lesion, invasion and metastasis (25). S100A6 was markedly expressed in cervical cancer tissues, and is predominantly expressed in fibroblasts and epithelial cells of the breast, heart, intestine, kidney, liver, ovary, placenta, stomach, thymus and uterus (26). Furthermore, increased expression of S100A6 has been identified in tumors (27). To the best of our knowledge, no previous studies have been performed that investigate the level of S100A6 in normal epithelial cell lines of cervix uteri. This was a limitation of the present study and it is therefore a recommendation for future studies in this field. The results of the present study demonstrated that S100A6 serves an important role in promoting the 


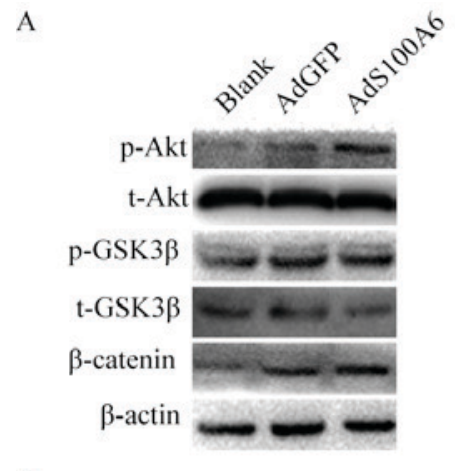

B

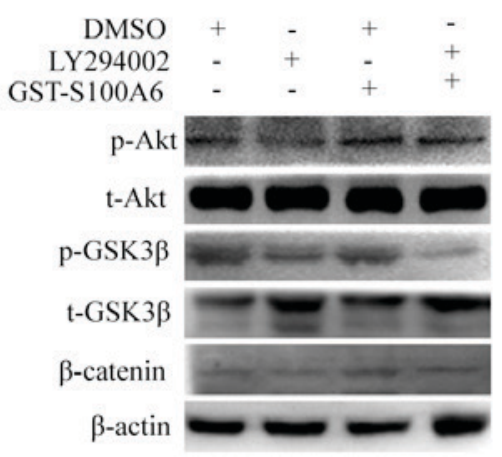

D

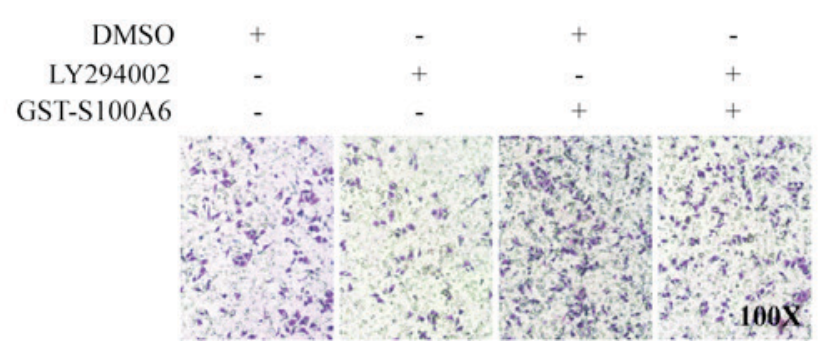

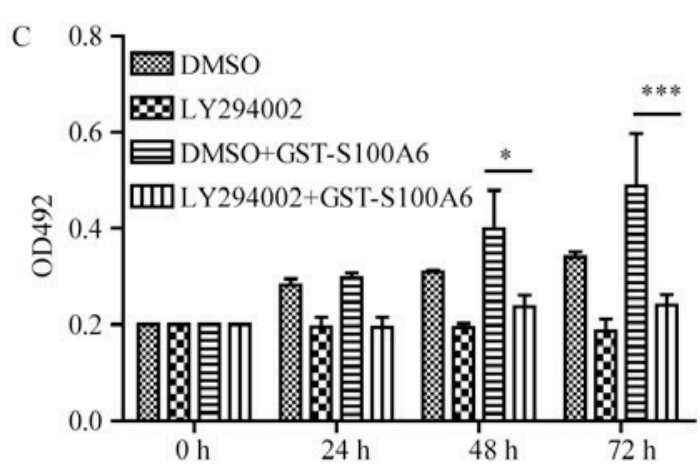

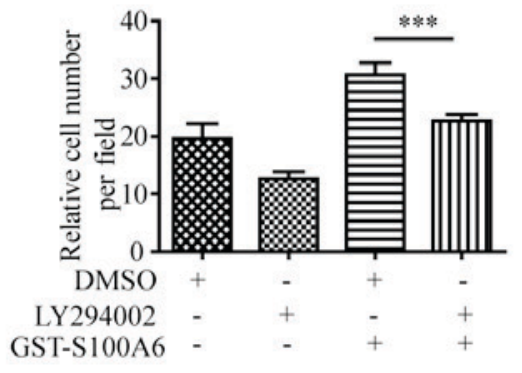

Figure 3. S100A6 activates the PI3K/Akt signaling pathway and promotes the viability and migration of cervical cancer cells. (A) HeLa cells were treated with AdS100A6 or AdGFP for $72 \mathrm{~h}$. Total Akt, total GSK3 $\beta$, $\beta$-catenin, p-Akt and p-GSK3 $\beta$ were detected using western blotting. (B) HeLa cells were treated with GST-S100A6 and LY294002 for $72 \mathrm{~h}$, and total Akt, total GSK3 $\beta$, $\beta$-catenin, p-Akt and p-GSK3 $\beta$ were detected using western blotting. (C) The effect of LY294002 on S100A6-enhanced proliferation of HeLa cells was determined using an MTT assay. HeLa cells were treated with GST-S100A6 (10 $\mu \mathrm{g} / \mathrm{ml})$ and LY294002 (10 $\mu \mathrm{M})$ for $72 \mathrm{~h}$. (D) The effect of LY294002 on S100A6-enhanced migration of HeLa cells was determined by a Transwell assay (magnification, x100). HeLa cells were treated with GST-S100A6 $(10 \mu \mathrm{g} / \mathrm{ml})$, LY294002 $(10 \mu \mathrm{M})$ or DMSO for $72 \mathrm{~h}$. The number of transmembrane cells were counted and calculated. All experiments were repeated three times. Results were presented as the mean \pm standard deviation. A one-way analysis of variance, followed by the Student-Newman-Keuls test was used to analyze the statistical significance of differences among groups. " $\mathrm{P}<0.05,{ }^{* * * *} \mathrm{P}<0.005, \mathrm{DMSO}+\mathrm{GST}-\mathrm{S} 100 \mathrm{~A} 6$ group compared with LY2940002 + GST-S100A6 group. p, phosphorylated; t, total; AdGFP, adenovirus carrying green fluorescent protein; AdS100A6, adenovirus carrying S100A6; OD, optical density; GST, glutathione transferase; DMSO, dimethyl sulfoxide.

proliferation and migration of cervical cancer cells through the PI3K/Akt signaling pathway.

HPV16 and HPV18 are the most common types of cervical cancer and account for $\sim 67 \%$ of all cervical carcinomas worldwide (28). HeLa cells are HPV $18^{+}$whereas SiHa cells are $\mathrm{HPV}_{16}{ }^{+}$, and HPV16 ${ }^{+}$and HPV18 ${ }^{+}$cervical cancer exhibit different biological behavior in vivo (29). This is due to the E6 and $\mathrm{E} 7$ gene products of HPV contributing to the pathogenesis of cancer. HPV integrates with the DNA within the host cell nucleus and thereby dysregulates the expression of the oncoproteins E6 and E7. Degradation of p53 is induced by E6, which results in a loss of p53 activity. Its degradation is accomplished through the formation of a complex with p53, E6 and E6-associated protein (30). In a physiological state, p53 functions to arrest cells in the $G_{1}$ phase of the cell cycle and induces apoptosis to allow the repair of host DNA (30). Furthermore, E7 binds to the cyclin-dependent kinase inhibitor, which results in a loss of control over the cell cycle (30). HeLa and SiHa are two different cell types, with different types of HPV; this is a potential explanation for why, in the present study, the S100A6 levels varied between the two cell lines.

In the present study, cervical cancer cells were treated with AdS100A6 and AdsiS100A6 to upregulate and downregulate the level of S100A6. The results of the present study suggest that S100A6 promotes the proliferation of cervical cancer cells, and these results were consistent with those of previous studies in colorectal cancer $(7)$, hepatocellular carcinoma $(8,31)$ and osteosarcoma (9). A Hoechst staining assay was performed to provide a visual result; however, there are human errors associated with the manual analysis and counting of cells. Consequently, the absence of flow cytometric data to detect apoptosis is a limitation of the present study. Furthermore, S100A6 was demonstrated to significantly promote the migration of cervical cancer cells, which is in accordance with a previous study on pancreatic duct adenocarcinoma (10). These results imply that S100A6 facilitates the metastasis of cervical cancer cells, as cell invasion and migration are the basic steps for tumor metastasis. Therefore, S100A6 may contribute to cervical cancer metastasis and may be a potential target for the treatment of cervical cancer, in order to increase the survival period of patients. However, the absence of data regarding the regulatory effect of S100A6 on cell invasion is a limitation of the present study.

A number of studies have reported that EMT has become a principal factor for tumor malignancy, as EMT contributes to the motility and invasiveness of tumor cells, and therefore contributes to distant metastasis (32-34). Cancer-associated EMT is a complicated and comprehensive reprogramming process that is involved in the metabolism, epigenetics and differentiation of cells (35). Previous studies have demonstrated that claudin-1, oncostatin-M receptor and lysine-specific demethylase 1 are associated with EMT and a poor outcome in cervical cancer (36-38). The results of the present study confirmed that S100A6 induced EMT in cervical cancer cells. Therefore, EMT serves an important role in the progression of cervical cancer. HeLa cells expressed lower levels of S100A6 and SiHa cells expressed higher levels of S100A6. Consequently, an increased level of S100A6 was 
attributed to the higher mesenchymal properties of SiHa cells. The level of E-cadherin was increased in HeLa cells compared with that of SiHa cells, whereas the level of N-cadherin was the opposite. These are consistent with the hypothesis that a function of S100A6 is to induce EMT in cervical cancer cells. In the present study, the expression of these molecules were determined by western blotting or semi-qPCR. A limitation of the present study is that the expression of these molecules was determined only at the mRNA or protein level.

A number of studies have demonstrated the involvement of S100A6 in a number of signaling pathways in many types of cancer cell (7-14). In cervical cancer cells, a number of pathways are implicated in cellular proliferation, apoptosis, migration and invasion, including $\mathrm{Wnt} / \beta$-catenin, mTOR and ERK signaling (39-42). In the present study, the PI3K/Akt signaling pathway was selected to determine the effect of S100A6 on cell proliferation and migration of cervical cancer cells. This is due to the PI3K/Akt signaling pathway serving an important role in tumorigenesis and the regulation of critical cellular functions, including survival, proliferation and metabolism (43). An increasing amount of evidence has demonstrated that the PI3K/Akt pathway is involved in the proliferation, apoptosis and autophagy of cervical cancer cells (44-46). It has been reported that Akt and matrix metalloproteinase 3 are key regulators of EMT in cervical cancer (47). It has also been suggested that the PI3K/Akt signaling pathway promotes metastasis and EMT in nasopharyngeal carcinoma (48). In our previous study, it was demonstrated that S100A6 may promote the proliferation and migration of osteosarcoma cells via the PI3K/Akt signaling pathway (49). Therefore, an aim of the present study was to determine whether the PI3K/Akt signaling pathway was also involved in S100A6-enhanced proliferation and migration of cervical cancer cells. GST-S100A6 and LY294002 were chosen to further validate the involvement of PI3K/Akt activation in S100A6-enhanced proliferation and migration of cervical cancer cells. The results demonstrated that S100A6 activated the PI3K/Akt signaling pathway and inhibition of the PI3K/Akt pathway markedly impaired S100A6-enhanced proliferation and migration of HeLa cells. Similar experiments were performed using AdS100A6; however, when cells were co-treated with AdS100A6 and LY294002, a large cohort of cells immediately underwent apoptosis due to the toxicity of polybrene and DMSO. Consequently, GST-S100A6 was used as it has a similar effect to AdS100A6 and is less toxic (50). Receptor for advanced glycation end-products (RAGE), Toll-like receptor 4 and epidermal growth factor receptors are receptors of the S100 protein, and S100A6 activates RAGE through distinct signal transduction pathways (51-53). Previous research of the present study cohort has demonstrated that S100A6 is an exocrine protein that can be secreted to the extracellular space, and the secreted S100A6 protein in the cell culture medium upregulated the mRNA level of endogenous S100A6 through Wnt/ $\beta$-catenin signaling. This subsequently affected the biological characteristics of tumor cells (50) and GST-S100A6 was demonstrated to act similarly to AdS100A6 (50). The secreted S100A6 protein was able to promote the proliferation and migration of colorectal cancer cells (50). Further research is required to explore the effects of LY294002 on EMT. Collectively, these results suggest that
S100A6 promotes the proliferation and migration of cervical cancer cells through the PI3K/Akt signaling pathway.

In conclusion, the results of the present study demonstrate that S100A6 serves a pivotal role in the malignancy of cervical cancer cells, including promoting the proliferation, migration and EMT of cervical cancer cells by activating the PI3K/Akt signaling pathway. The present study provides evidence to improve the understanding of the pathophysiological process of cervical cancer as S100A6 may be a promising molecular target for patients with cervical cancer. However, the absence of in vivo data and data regarding the expression and clinical significance of S100A6 in cervical cancer tissues are limitations of the present study. Therefore, further in vivo studies and data regarding the expression and clinical significance of S100A6 in cervical cancer tissues based on in vivo studies and databases including Oncomine are required to verify these issues and confirm the role of S100A6 as a novel biomarker for the detection and therapy of cervical cancer.

\section{Acknowledgements}

The authors would like to thank Dr T.C. He (Medical Center, The University of Chicago, Chicago, IL, USA) for the gifts of AdS100A6, AdsiS100A6, AdGFP and AdRFP.

\section{Funding}

The present study was supported by the Chongqing Graduate Student Research Innovation Project Funding (grant no. CYS15133) and Chongqing Yuzhong District Science and Technology Project Funding (Basic and Frontier Research) (grant no. 20160106).

\section{Availability of data and materials}

The datasets used and/or analyzed during the present study are available from the corresponding author on request.

\section{Authors' contributions}

AL performed most of the research and was a major contributor in writing the manuscript. YG, XL, HS, JX, JZ, MH, LC and QP prepared experimental materials and reviewed the article. HZ, YZ, YW and LZ made substantial contributions to the design of the work, drafting the manuscript or revising it critically for important intellectual content. LZ gave final approval of the version to be published.

\section{Ethics approval and consent to participate}

Not applicable.

\section{Consent for publication}

Not applicable.

\section{Competing interests}

The authors declare that they have no competing interests. 


\section{References}

1. Liu C, Lin J, Li L, Zhang Y, Chen W, Cao Z, Zuo H, Chen C and Kee K: HPV16 early gene E5 specifically reduces miRNA-196a in cervical cancer cells. Sci Rep 5: 7653, 2015.

2. zur Hausen H: Papillomaviruses and cancer: From basic studies to clinical application. Nat Rev Cancer 2: 342-350, 2002.

3. Das BC, Hussain S, Nasare V and Bharadwaj M: Prospects and prejudices of human papillomavirus vaccines in India. Vaccine 26: 2669-2679, 2008.

4. Heizmann CW, Fritz G and Schafer BW: S100 proteins: Structure, functions and pathology. Front Biosci 7: 1356-1368, 2002.

5. An G, Xu Y, Shi L, Shizhen Z, Deng S, Xie Z, Sui W, Zhan F and Qiu L: Chromosome 1q21 gains confer inferior outcomes in multiple myeloma treated with bortezomib but copy number variation and percentage of plasma cells involved have no additional prognostic value. Haematologica 99: 353-359, 2014.

6. Ha K, Shen Y, Graves T, Kim CH and Kim HG: The presence of two rare genomic syndromes, 1q21 deletion and Xq28 duplication, segregating independently in a family with intellectual disability. Mol Cytogenet 9: 74, 2016.

7. Duan L, Wu R, Zou Z, Wang H, Ye L, Li H, Yuan S, Li X, Zha H, Sun $\mathrm{H}$, et al: S100A6 stimulates proliferation and migration of colorectal carcinoma cells through activation of the MAPK pathways. Int J Oncol 44: 781-790, 2014.

8. Li Z, Tang M, Ling B, Liu S, Zheng Y, Nie C, Yuan Z, Zhou L, Guo G, Tong A and Wei Y: Increased expression of S100A6 promotes cell proliferation and migration in human hepatocellular carcinoma. J Mol Med (Berl) 92: 291-303, 2014.

9. Li Y, Wagner ER, Yan Z, Wang Z, Luther G, Jiang W, Ye J, Wei Q, Wang J, Zhao L, et al: The Calcium-Binding Protein S100A6 accelerates human osteosarcoma growth by promoting cell proliferation and inhibiting osteogenic differentiation. Cell Physiol Biochem 37: 2375-2392, 2015.

10. Chen X, Liu X, Lang H, Zhang S, Luo Y and Zhang J: S100 calcium-binding protein A6 promotes epithelial-mesenchymal transition through $\beta$-catenin in pancreatic cancer cell line. PloS One 10: e0121319, 2015.

11. Wang XH, Zhang LH, Zhong XY, Xing XF, Liu YQ, Niu ZJ, Peng Y, Du H, Zhang GG, Hu Y, et al: S100A6 overexpression is associated with poor prognosis and is epigenetically up-regulated in gastric cancer. Am J Pathol 177: 586-597, 2010.

12. Kilańczyk E, Graczyk A, Ostrowska H, Kasacka I, Leśniak W and Filipek A: S100A6 is transcriptionally regulated by $\beta$-catenin and interacts with a novel target, lamin $\mathrm{A} / \mathrm{C}$, in colorectal cancer cells. Cell Calcium 51: 470-477, 2012.

13. Li A, Shi D, Xu B, Wang J, Tang YL, Xiao W, Shen G, Deng W and Zhao C: S100A6 promotes cell proliferation in human nasopharyngeal carcinoma via the p38/MAPK signaling pathway. Mol Carcinog 56: 972-984, 2017.

14. Joo JH, Kim JW, Lee Y, Yoon SY, Kim JH, Paik SG and Choe IS: Involvement of NF-kappaB in the regulation of S100A6 gene expression in human hepatoblastoma cell line HepG2. Biochem Biophys Res Commun 307: 274-280, 2003.

15. Robbins HL and Hague A: The PI3K/Akt pathway in tumors of endocrine tissues. Front Endocrinol (Lausanne) 6: 188, 2016.

16. Saji M, Vasko V, Kada F, Allbritton EH, Burman KD and Ringel MD: Akt1 contains a functional leucine-rich nuclear export sequence. Biochem Biophys Res Commun 332: 167-173, 2005.

17. Hers I, Vincent EE and Tavaré JM: Akt signalling in health and disease. Cell Signal 23: 1515-1527, 2011.

18. Martelli AM, Tabellini G, Bressanin D, Ognibene A, Goto K, Cocco L and Evangelisti C: The emerging multiple roles of nuclear Akt. Biochim Biophys Acta 1823: 2168-2178, 2012.

19. Ocana A, Vera-Badillo F, Al-Mubarak M, Templeton AJ, Corrales-Sanchez V, Diez-Gonzalez L, Cuenca-Lopez MD, Seruga B, Pandiella A and Amir E: Activation of the $\mathrm{PI} 3 \mathrm{~K} / \mathrm{mTOR} / \mathrm{AKT}$ pathway and survival in solid tumors: Systematic review and meta-analysis. PloS One 9: e95219, 2014.

20. Santamaria PG, Moreno-Bueno G, Portillo F and Cano A: EMT: Present and future in clinical oncology. Mol Oncol 11: 718-738, 2017.

21. Miao JK, Lai TX, Zuo GW, et al: Expression and purification of human S100A6-GST fusion protein. J Chongqing Med Univ 32: 1009-1012, 2007.

22. Duan L, Wu R, Ye L, Wang H, Yang X, Zhang Y, Chen X, Zuo G, Zhang Y, Weng Y, et al: S100A8 and S100A9 are associated with colorectal carcinoma progression and contribute to colorectal carcinoma cell survival and migration via $\mathrm{Wnt} / \mathrm{b}$-catenin pathway. PloS One 8: e62092, 2013.
23. Song Q, Zhong L, Chen C, Tang Z, Liu H, Zhou Y, Tang M, Zhou L, Zuo G, Luo J, et al: miR-21 synergizes with BMP9 in osteogenic differentiation by activating the BMP9/Smad signaling pathway in murine multilineage cells. Int J Mol Med 36: 1497-1506, 2015.

24. Nedjadi T, Kitteringham N, Campbell F, Jenkins RE, Park BK, Navarro P, Ashcroft F, Tepikin A, Neoptolemos JP and Costello E: S100A6 binds to annexin 2 in pancreatic cancer cells and promotes pancreatic cancer cell motility. Br J Cancer 101: $1145-1154,2009$

25. Yuan T: The expression of tumour metastasis-associated genes S100A6, Rab5a in cervical cancer and its clinical significance. Guangxi Medical University, 2010.

26. Kuźnicki J, Kordowska J, Puzianowska M and Woźniewicz BM: Calcyclin as a marker of human epithelial cells and fibroblasts. Exp Cell Res 200: 425-430, 1992.

27. Lesniak W, Slomnicki LP and Filipek A: S100A6-new facts and features. Biochem Biophys Res Commun 390: 1087-1092, 2009.

28. Xiao M, Xu Q, Li H, Gao H, Bie Y and Zhang Z: Prevalence of human papillomavirus genotypes among women with high-grade cervical lesions in Beijing, China. Medicine (Baltimore) 95: e2555, 2016.

29. Schwartz SM, Daling JR, Shera KA, Madeleine MM, McKnight B, Galloway DA, Porter PL and McDougall JK: Human papillomavirus and prognosis of invasive cervical cancer: A population-based study. J Clin Oncol 19: 1906-1915, 2001.

30. Bansal A, Singh MP and Rai B: Human papillomavirus-associated cancers: A growing global problem. Int J Appl Basic Med Res 6: 84-89, 2016.

31. Hua Z, Chen J, Sun B, Zhao G, Zhang Y, Fong Y, Jia Z and Yao L: Specific expression of osteopontin and S100A6 in hepatocellular carcinoma. Surgery 149: 783-791, 2011.

32. Bedi U, Mishra VK, Wasilewski D, Scheel C and Johnsen SA: Epigenetic plasticity: A central regulator of epithelial-to-mesenchymal transition in cancer. Oncotarget 5: 2016-2029, 2014.

33. Baum B, Settleman J and Quinlan MP: Transitions between epithelial and mesenchymal states in development and disease. Semin Cell Dev Biol 19: 294-308, 2008

34. Thiery JP, Acloque H, Huang RY and Nieto MA: Epithelial-mesenchymal transitions in development and disease. Cell 139: 871-890, 2009.

35. Li L and Li W: Epithelial-mesenchymal transition in human cancer: Comprehensive reprogramming of metabolism, epigenetics, and differentiation. Pharmacol Ther 150: 33-46, 2015.

36. Zhang WN, Li W, Wang XL, Hu Z, Zhu D, Ding WC, Liu D, Li KZ, Ma D and Wang H: CLDN1 expression in cervical cancer cells is related to tumor invasion and metastasis. Oncotarget 7: 87449-87461, 2016.

37. Kucia-Tran JA, Tulkki V, Smith S, Scarpini CG, Hughes K, Araujo AM, Yan KY, Botthof J, Pérez-Gómez E, Quintanilla M, et al: Overexpression of the oncostatin-M receptor in cervical squamous cell carcinoma is associated with epithelial-mesenchymal transition and poor overall survival. Br J Cancer 115: 212-222, 2016

38. Liu Y, Wang Y, Chen C, Zhang J, Qian W, Dong Y, Liu Z, Zhang X, Wang X, Zhang Z, et al: LSD1 binds to HPV16 E7 and promotes the epithelial-mesenchymal transition in cervical cancer by demethylating histones at the Vimentin promoter. Oncotarget 8: 11329-11342, 2016.

39. Chen Q, Cao HZ and Zheng PS: LGR5 promotes the proliferation and tumor formation of cervical cancer cells through the Wnt/ $\beta$-catenin signaling pathway. Oncotarget 5: 9092-9105, 2014.

40. Kong L, Hao Q, Wang Y, Zhou P, Zou B and Zhang YX: Regulation of $\mathrm{p} 53$ expression and apoptosis by vault RNA2-1-5p in cervical cancer cells. Oncotarget 6: 28371-28388, 2015.

41. Rashmi R, DeSelm C, Helms C, Bowcock A, Rogers BE, Rader JL, Grigsby PW and Schwarz JK: AKT inhibitors promote cell death in cervical cancer through disruption of mTOR signaling and glucose uptake. PloS One 9: e92948, 2014.

42. Liu T, Liu Y, Bao X, Tian J, Liu Y and Yang X: Overexpression of TROP2 predicts poor prognosis of patients with cervical cancer and promotes the proliferation and invasion of cervical cancer cells by regulating ERK signaling pathway. PloS One 8: e75864, 2013.

43. Yang SX, Polley E and Lipkowitz S: New insights on PI3K/AKT pathway alterations and clinical outcomes in breast cancer. Cancer Treat Rev 45: 87-96, 2016.

44. Feng T, Zheng L, Liu F, Xu X, Mao S, Wang X, Liu J, Lu Y, Zhao W, Yu X and Tang W: Growth factor progranulin promotes tumorigenesis of cervical cancer via PI3K/Akt/mTOR signaling pathway. Oncotarget 7: 58381-58395, 2016. 
45. Qian S and Li M: Chamaejasmine induces apoptosis in HeLa cells through the PI3K/Akt signaling pathway. Anticancer Drugs 28: 40-50, 2017.

46. Jeyamohan S, Moorthy RK, Kannan MK and Arockiam AJ: Parthenolide induces apoptosis and autophagy through the suppression of PI3K/Akt signaling pathway in cervical cancer. Biotechnol Lett 38: 1251-1260, 2016.

47. Hagemann T, Bozanovic T, Hooper S, Ljubic A, Slettenaar VI, Wilson JL, Singh N, Gayther SA, Shepherd JH and Van Trappen PO: Molecular profiling of cervical cancer progression. Br J Cancer 96: 321-328, 2007.

48. Ke LR, Xiang YQ, Guo X, Lu J, Xia W, Yu Y, Peng Y, Wang L, Wang G, Ye Y, et al: c-Src activation promotes nasopharyngeal carcinoma metastasis by inducing the epithelial-mesenchymal transition via PI3K/Akt signaling pathway: A new and promising target for NPC. Oncotarget 7: 28340-28355, 2016.

49. Wang H, Zou Z, Duan L, Chen X, Li H, Yuan S, He TC and Zhou L: S100A6 promotes proliferation and migration of human osteosarcoma cell line 143B through PI3K/Akt signaling pathway. Chin J Pathophysiol 29: 1928-1933, 2013.
50. Hui S: Exogenous S100A6 upregulates its own expression in colorectal carcinoma cells and its mechanism. Chongqing Medical University, 2013.

51. Sparvero LJ, Asafu-Adjei D, Kang R, Tang D, Amin N, Im J, Rutledge R, Lin B, Amoscato AA, Zeh HJ and Lotze MT: RAGE (Receptor for Advanced Glycation Endproducts), RAGE ligands, and their role in cancer and inflammation. J Transl Med 7: 17, 2009.

52. Chen H, Xu C, Jin Q and Liu Z: S100 protein family in human cancer. Am J Cancer Res 4: 89-115, 2014.

53. Bresnick AR, Weber DJ and Zimmer DB: S100 proteins in cancer. Nat Rev Cancer 15: 96-109, 2015.

cc) (i) $\ominus$ This work is licensed under a Creative Commons cc) Attribution-NonCommercial-NoDerivatives 4.0 International (CC BY-NC-ND 4.0) License. 